\title{
CLINICAL AND PATHOLOGICAL FINDINGS IN OCHRONOSIS
}

\author{
BY \\ LOYD R. WAGNER, JAMES L. KNOTT, REGINALD A. MACHAFFIE, \\ AND JOHN R. WALSH \\ From the Veterans Administration Hospital, Omaha, Nebraska
}

\begin{abstract}
A case of ochronosis with far-advanced cardiovascular, skeletal, and prostatic manifestations is reported. An attempt is made to correlate the location of pigment with other pathological changes in this disorder. A brief discussion of the hereditary factors, the metabolic pathways of homogentisic acid, and the pathogenesis of ochronosis is presented.
\end{abstract}

Ochronosis frequently presents as a triad of arthritis, dark urine, and bluish discoloration of cartilage. Angina is a late manifestation, and necropsies have demonstrated considerable atherosclerotic changes in the intima and media of the coronary arteries (Lichtenstein and Kaplan, 1954). The following case seems worthy of presentation because of the unusual aspects of coronary thrombosis and prostatic calcification, which clinically resembled carcinoma of the prostate. In addition the pathological distribution of pigment suggested certain theoretical considerations concerning the deposition of homogentisic acid which will be discussed.

\section{Case Report (No. 15008)}

A 54-year-old white man was admitted to the hospital on August 4, 1956, because of increasing exertional chest pain and dyspnoea.

He had noted that his underclothing had been stained with black deposits from his urine since childhood, and, in 1937, a peculiar discoloration of the ears and sclera was observed. At the age of 29 he-spent a short time in hospital because of a back injury. He was thereafter asymptomatic until 1941 when he developed persistent low back pain radiating into the right leg which was aggravated by jarring movements. In 1945 he was examined for arthritis of the back and legs, diagnosed as having ochronosis, and was medically retired from the armed forces. Because of ankylosis of the right knee, he was in hospital in 1950, at which time his right leg was treated with casting and arthroplasty. A three months' course of A.C.T.H. gave partial relief.

In November, 1954, he was first admitted to the Veterans Administration Hospital because of pain and stiffness in the neck, shoulders, and arms, and an exacerbation of the right leg and back pain. The blood pressure was $164 / 110 \mathrm{~mm}$. Hg. The diagnosis of alkaptonuria with ochronosis was confirmed. Because the prostate was enlarged, hard, asymmetrical, painless, and fixed, a needle biopsy was necessary and revealed ochronotic calculi with no evidence of tumour. Subsequently during cystoscopy a large, irregular, black, staghorn calculus was seen in the prostatic urethra and removed. Furthermore, transurethrally resected prostatic specimens contained numerous, small, black calculi within acini which were consistent with ochronotic involvement of the prostate with no evidence of carcinoma. In March, 1955, a calculus was removed from the tip of the urethra by meatotomy.

In June, 1955, he first developed exertional dyspnoea and chest pain which radiated into the shoulders and arms. He also noted nocturnal dyspnoea. On September 21, 1955, he was awakened by a severe, crushing, precordial chest pain radiating into both shoulders and down the ulnar aspects of the arms, associated with dyspnoea and diaphoresis. This pattern recurred the following day, requiring the patient's admission to the hospital, where electrocardiographic evidence of acute myocardial infarction was obtained. The serum glutamic oxalacetic transaminase level was 112 units. The course was uneventful except for thrombophlebitis of the left leg, and he was discharged on December 22, 1955, on digitalis, dietary sodium restrictions, vasodilator drugs, and $25 \mathrm{mg}$. of bis-hydroxycoumarin per day. Subsequently he had angina of effort three to four times a day, attacks which increased during the following winter months. On April 17, 1956, he was again admitted to hospital because of increasing chest pain, orthopnoea, and a moderate cough.

Physical Examination.-The patient was a short, fairly well-developed, obese, white male who appeared chronically ill. The aural pinnae and ridge of the nose were bluish-grey. The lateral portions of the sclerae contained dark brown pigmentation. Ophthalmoscopic examination revealed moderate AV-nicking and arteriolar attenuation and tortuosity. 
The neck was hyperextended and there was marked limitation of all cervical movement. The thoracic cage was fixed in inspiration with marked dorsal kyphosis, slight dextroscoliosis, and increased anteroposterior diameter. The blood pressure was $130 / 90$ $\mathrm{mm}$. Hg with a regular heart rate of 92 per minute. The point of maximum impulse was displaced to the left and there was a Grade II, blowing, systolic, apical murmur transmitted into the aortic area. The prostate was hard, fixed, asymmetrical, and not tender. Popliteal and dorsalis pedis arterial pulsations were palpable. The lower extremities were atrophic, markedly deformed, and limited in movements.

Laboratory Findings.-In the course of his several admissions, the total blood count, erythrocyte sedimentation rate, Kolmer, serum albumin and globulin, serum cholesterol and esters, glucose tolerance, cephalin cholesterol flocculation, alkaline and acid phosphatase, inorganic phosphorus, blood urea nitrogen, and serum uric acid had been within normal limits. Urine analyses were acid in reaction, with specific gravities up to 1.023 with normal urinary sediments. On standing, the urine became progressively brown and turned black on alkalinization. Quantitative analysis revealed $4.3 \mathrm{~g}$.

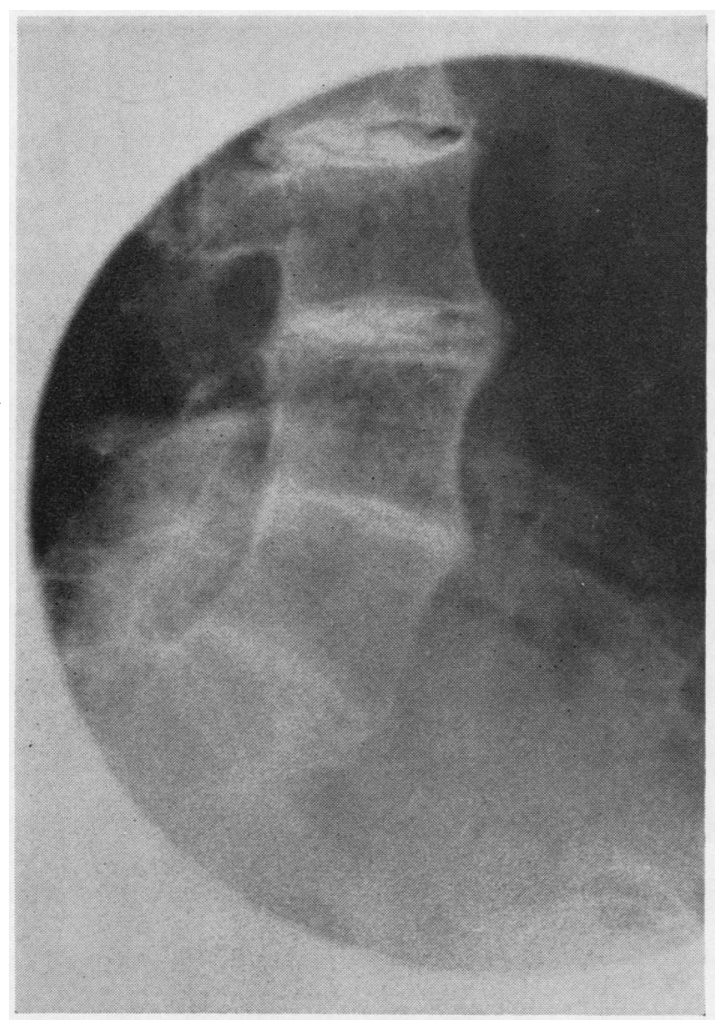

Fig. 1.-Lateral view of the lumbo-sacral spine showing narrowed joint spaces, osteoporotic vertebral bodies, and marked calcification of the intervertebral discs and ligaments. of homogentisic acid per litre of urine. Cerebrospinal fluid total protein was $59 \mathrm{mg} . \%$.

Radiographs revealed marked degenerative changes in the knees, sacro-iliac, hip, and shoulder joints. Films of the thoraco-lumbar spine demonstrated osteoporotic vertebral bodies and marked calcification of the intervertebral ligaments and of the intervertebral discs, findings which were thought to be diagnostic of ochronotic arthritis (Fig. 1).

An electrocardiogram showed changes of an acute anterior inferior myocardial infarction on September 26 , 1955, with evolutionary changes noted on subsequent tracings.

During the final admission the patient was placed at bed rest on a sodium-restricted diet and given digitalis, vasodilators, and anticoagulant therapy. Transitory improvement was followed on April 19, 1956, by cardiac asystole and sudden death despite emergency treatment.

Necropsy Findings.-External examination of the body revealed arthritic deformity of all the joints. The skin was normal except over cartilaginous areas, where blue-black discoloration was visible. Dissection of the musculature from the chest wall revealed blackish discoloration of the costal cartilages (Fig. 2).

The heart (weight, 550 g.) revealed calcific atheromatous plaques in the aortic and mitral valve cusps and similar plaques throughout the aorta and coronary arteries. These plaques were blue-black and similar pigmentary changes occurred in the chordae tendineae. There was old and recent infarction of the anterior wall of the left ventricle, the anterior portion of the interventricular septum, and the apex of the heart secondary to thrombosis of the anterior-descending branch of the left coronary artery (Fig. 3).

The lungs were oedematous and congested. The tracheal and bronchial cartilages were discoloured, mainly at the periphery.

The surfaces of the kidneys were pitted and irregular. On section, the medulla was reddish-black and the arcuate arteries were outlined by black pigment. The urine in the bladder was dark and the mucosa was bluish-black. Black calculi were present in grossly dilated acini of a hard and fibrous prostate.

The bony trabeculae of the sternal marrow, the intervertebral disc cartilages, the cartilage and synoviae of the knee joint, and the quadratus femoris tendon were black and brittle (Fig. 4).

The vessels in the dura and the brain were outlined by black pigment deposits.

Microscopy of the heart revealed an acute infarct superimposed on areas of fibrosis. Sections of the coronary arteries and aorta revealed brown, granular pigment in the endothelial cells and also in calcified atheromatous plaques (Fig. 5). Similar endothelial and perivascular pigmentation was seen in the lungs and other viscera with endothelial pigmentation, especially marked in the spleen, adrenals, the arcuate arteries of the kidneys, and submucosal vessels of the 


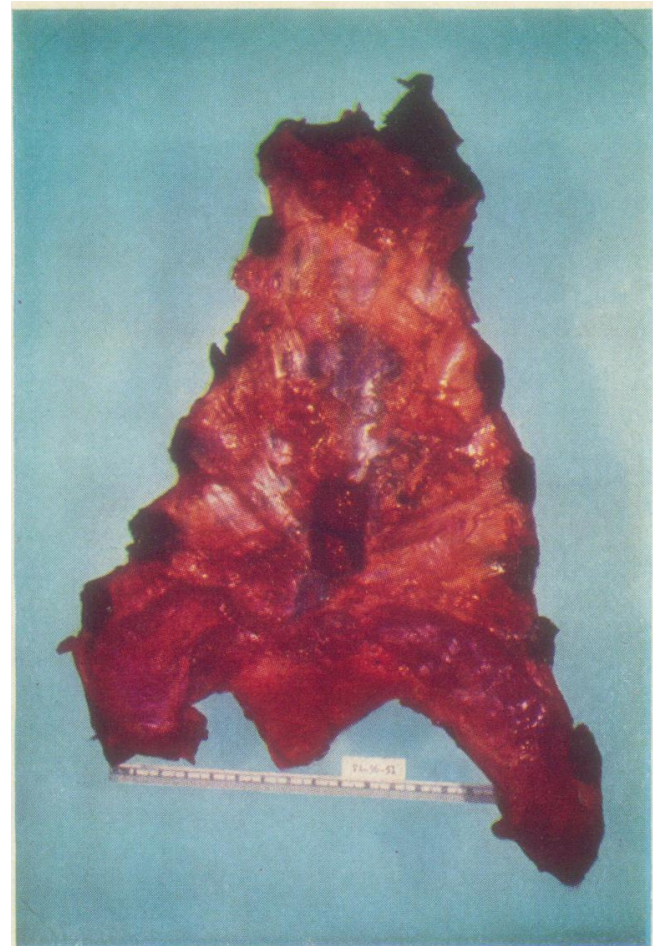

Fig. 2.-Under-surface of the sternum. The costochondral cartilages are markedly pigmented.

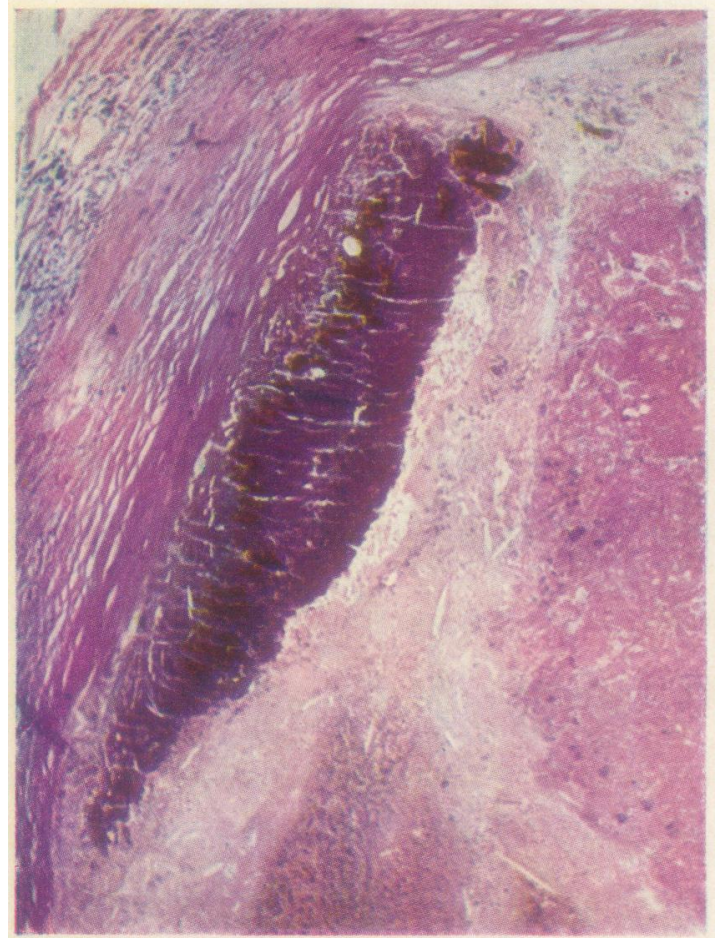

FIG. 5.-Section of the occluded artery $\times 150$. A pigmented atheromatous plaque is located at the site of the occluding thrombus.

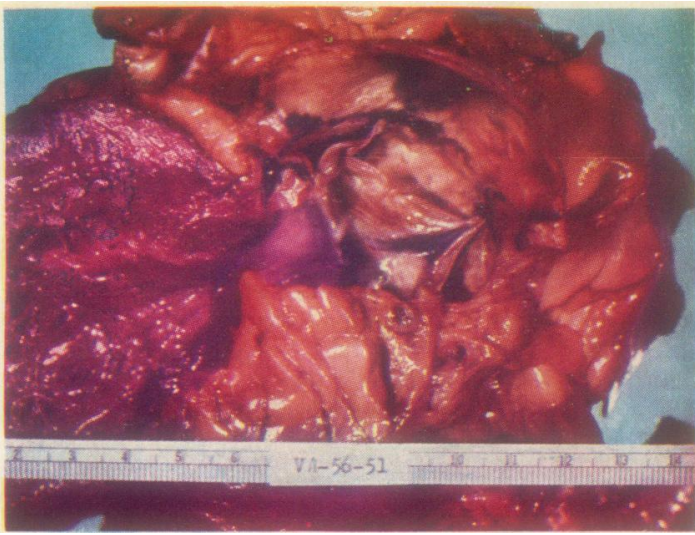

Fig. 3.-Pigmented areas are seen in the thrombus of the coronary vessels and in the region of the aortic valves.

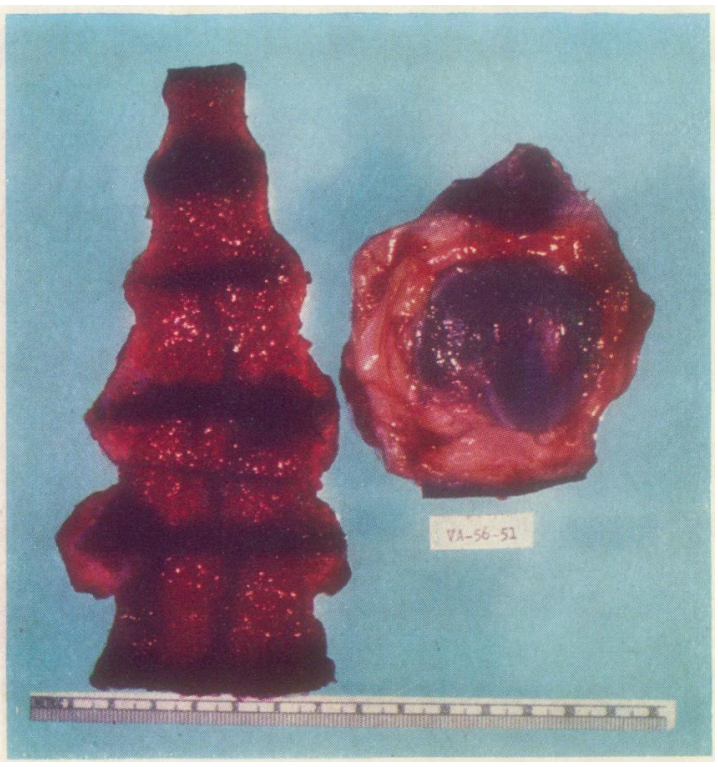

FIG. 4. - Sagittal section of the lumbosacral vertebra. On the right is a view of the articular surface of the right knee joint, which shows similar pigmentation.

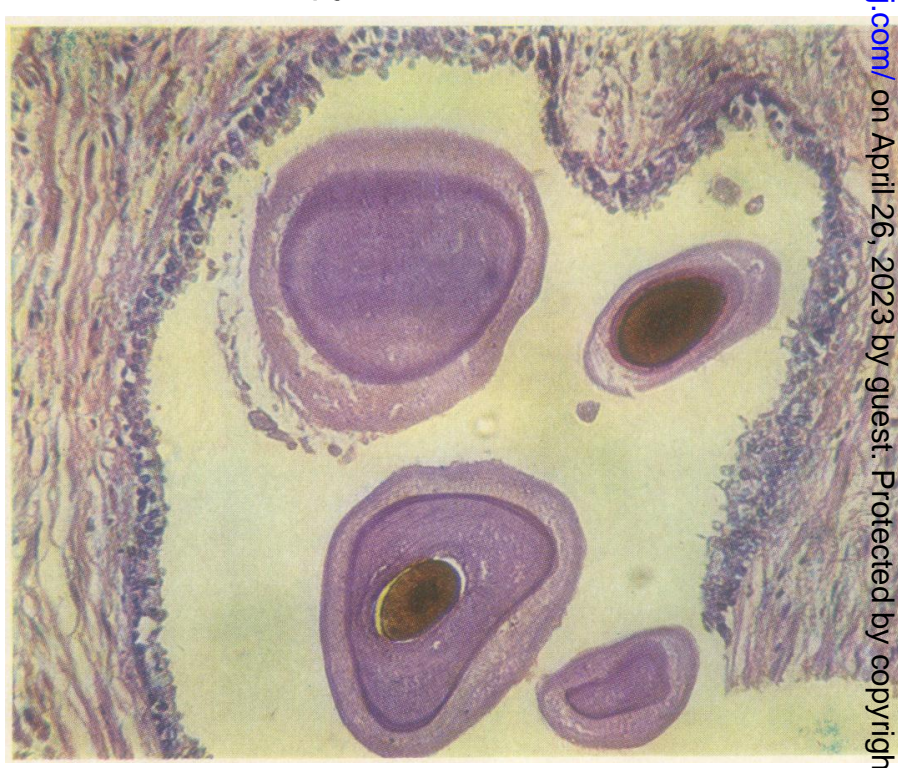

Fig. 6. - Section of prostatic acinus $\times 150$. The dilated acinus contains pigmented calculi and corpora a m ylacea. 
urinary bladder. In the perivascular tissue, granular pigment was prominent and occurred both within histiocytes and free in the extracellular spaces. Liver parenchymal cells were pigmented, as was renal tubular epithelium.

The prostate was fibrous and pigmented calculi and corpora amylacea were numerous within dilated acini (Fig. 6).

The villous synovium of the knee showed fibrosis and calcification occurring about extracellular brown pigment granules. Here again, endothelial cells were heavily pigmented. In skeletal structures, the heaviest pigmentation occurred beneath periosteum, perichondrium, and in those vascular areas subject to trauma. Cartilage and bone architecture, however, remained essentially normal despite pigmentation.

\section{Discussion}

Pathologically it appears that there are two primary sites of pigment deposition in this patient with ochronosis, namely vascular endothelium and the organs of excretion. Liberation of granules from the endothelial cells may be capable of causing the degenerative changes seen in this disorder of metabolism. These include the hypertrophic arthritis and atherosclerosis. Excretion of homogentisic acid accounts for pigmentation in many other sites such as liver, sweat glands, renal tubules, prostatic acini, and bladder mucosa.

The pathological findings in this patient with ochronosis correlate with those previously documented in the medical literature (Lichtenstein and Kaplan, 1954 ; La Du, Seegmiller, Laster, and Zannoni, 1958a and b; Haldane, 1941; Galdston, Steele, and Dobriner, 1952 ; Garrod, 1908, 1923 ; Oppenheimer and Kline, 1922). However, the usual changes in the skin due to pigment deposits in endothelial cells of the dermal vessels and epithelial cells of sweat glands (Lichtenstein and Kaplan, 1954) were not found in our patient.

The patient showed the cardiovascular pigmentation in the endothelial cells, intima, media, and perivascular tissues (Lichtenstein and Kaplan, 1954; Oppenheimer and Kline, 1922) as previously reported. These changes appear to accentuate atherosclerosis. In fact, roughened intimal plaques were the site of thrombus formation and were the cause of myocardial infarction.

Cartilage pigmentation and secondary hypertrophic arthritic changes were especially prominent in the knees, spine, and shoulders, and were similar to those reported elsewhere in the literature (Sutro and Anderson, 1947 ; Kolaczek, 1910 ; Pomeranz, Friedman, and Tunick, 1941).
In cartilage the greatest deposits occur peripherally beneath the perichondrium. With pigmentation of the cartilage, it becomes brittle and breaks when submitted to stress, leading to traumatic arthritis due to free joint bodies. Synovial reaction occurs secondarily to trauma and some fibrosis also in the synovial tissues in response to extracellular granular pigment.

Pigmentation of arcuate arterial endothelium and also of renal tubular epithelium has been documented previously (Galdston et al., 1952), and also occurs in this case. Prostatic calculi and corpora amylacea are pigmented and appear to result from excretion of homogentisic acid by this organ (Young, 1944). The calculi and secondary fibrosis were responsible for the clinical findings suggestive of carcinoma. The need for biopsy proof of carcinoma is further substantiated, and this particular case illustrates the limits of digital examination.

Pigment has been reported to occur in various endocrine structures of the body, especially the pancreatic islets, pituitary gland, and the pineal body (Lichtenstein and Kaplan, 1954; Galdston et al., 1952 ; Garrod, 1908, 1923 ; Oppenheimer and Kline, 1922; Boyd, 1947).

Garnier and Voirin demonstrated that homogentisic acid, an intermediate metabolite of phenylalanine and tyrosine, is completely metabolized by normal individuals (Garnier and Voirin, 1892). Alkaptonurics were shown to have an inability to metabolize compounds at the 2,5 positions on the benzene ring of homogentisic acid. The metabolic defect of alkaptonuria (hereditary ochronosis) lies in this inability and is the result of a single recessive Mendelian factor (gene) mutation. This alters the ability of the cell to produce the enzyme (homogenticase, homogentisic oxidase) responsible for the degradatory oxidation of homogentisic acid to fumaryl acetoacetic acid (La Du et al., $1958 \mathrm{a}$ and b). Other mutative changes are involved in the failure to convert phenylalanine to melanin (albinism) ; inability to transpose the hydroxyl groups of 3,4 dihydroxyphenylalanine to the 2,5 positions (tyrosinosis) ; and the loss of the enzyme necessary to oxidize the $p$-hydroxypyruvic acid to the 2,5 dihydroxy form (phenylpyruvic oligophrenia).

Garrod (1908, 1923) first recognized and described the hereditary nature of ochronosis in 1904, and noted the high frequency of consanguineous marriages in family pedigrees. He explained the pathological findings by the concept of " inborn errors of metabolism," 
postulating a genetically controlled absence of an enzyme. Others have corroborated Garrod's findings (Hogben, Worrall, and Zieve, 1932).

The pathological changes seen in ochronosis have been thought, by previous authors (Lichtenstein and Kaplan, 1954 ; La Du et al., 1958a and b ; Haldane, 1941 ; Galdston et al., 1952; Garrod, 1908, 1923 ; Oppenheimer and Kline, 1922 ; Sutro and Anderson, 1947), to be the result of accumulation of homogentisic acid and its polymers in tissues such as cartilage, fibrocartilage, tendons, and the supportive fibrous tissues of the arteries and of the heart. The special affinity of these tissues was thought to be enhanced by the high concentration of homogentisic acid. Physical stress may conceivably cause injury to cells of vascular tissue with disruption of the cellular membrane and liberation of pigment granules, which may be capable of promoting degenerative changes in the adjacent perivascular spaces (Boyd, 1947). The presence of foreign material within a relatively anoxic tissue greatly enhances fibrosis. Contraction of the fibrous tissue may result in additional anoxia, tissue destruction, and progression of the pathological changes demonstrated in ochronosis. Such a theory of stress in an area of relatively poor vascularity can explain the changes which are so marked in the cartilages, tendons, and especially within the skeletal joint spaces.

Illustrations by Frank Roberts and John Friel, Medical Illustration Service, Veterans Administration Hospital, Omaha, Nebraska.

\section{REFERENCES}

Boyd, W. (1947). A Text Book of Pathology, 5th ed. Lea and Febiger, Philadelphia.

Galdston, M. Steele, J. Murray, and Dobriner, K. (1952), Amer. . J. Med., 13, 432 .

Garnier, L., and Voirin, G. (1892). Arch. Physiol. norm. Dath. 5 ser., 4,225

Garrod, A. E. (1908). Lancet, 2, 142.

- (1923). Inborn Errors of Metabolism, 2nd ed., p. 43. Oxford University Press, London.

Haldane, J. B. S. (1941). New Paths in Genetics. Allen and Unwin, London.

Hogben, L., Worrall, R. L., and Zieve, I. (1932). Proc. roy. Soc Edinb., 52, 264.

Kolaczek, H. (1910). Beitr. klin. Chir., 71, 254

La Du, B. N., Seegmiller, J. B., Laster, L., and Zannoni, V. (1958a). J. biol. Chem., 230, 251.

(1958b). Bull. rheum. Dis. 8 No. 9 (p. 163).

Lichtenstein, L., and Kaplan, L. (1954). Amer. J. Path., 30, 99.

Oppenheimer, B. S., and Kline, B. S. (1922). Arch. intern. Med., 29, 732.

Pomeranz, M. M., Friedman, L. J., and Tunick, I. S. (1941) Radiology, 37, 295.

Sutro, C. J., and Anderson, M. E. (1947). Surgery, 22, 120.

Young, H. H. (1944). J. Urol. (Baltimore), 51, 48. 